\title{
Inhibition of TRPA1 and IL-6 Signal Alleviates Neuropathic Pain Following Chemotherapeutic Bortezomib
}

\author{
Dongxue $\mathrm{LIU}^{1} *$, Mingming $\mathrm{SUN}^{1}{ }^{*}$, Dongsheng $\mathrm{XU}^{1}$, Xiaohui $\mathrm{MA}^{1}$, Dongmei $\mathrm{GAO}^{2}$, Hong $\mathrm{YU}^{2}$ \\ * These authors contributed equally to this work. \\ ${ }^{1}$ Tumor Center, The First Hospital of Jilin University, Changchun, Jilin, China, ${ }^{2}$ Department of \\ Otorhinolaryngology, The First Hospital of Jilin University, Changchun, Jilin, China
}

Received September 11, 2018

Accepted January 3, 2019

Epub Ahead of Print August 19, 2019

\begin{abstract}
Summary
Bortezomib (BTZ) is used as a chemotherapeutic agent for the treatment of multiple myeloma. Nevertheless, one of the significant limiting complications of BTZ is painful peripheral neuropathy during BTZ therapy. Thus, in this study we examined signaling pathways of interleukin-6 (IL-6) and transient receptor potential ankyrin 1 (TRPA1) in the sensory nerves responsible for neuropathic pain induced by BTZ and further determined if influencing the pathways can improve neuropathic pain. ELISA and western blot analysis were used to examine the levels of IL-6, and IL-6 receptor (IL-6R), TRPA1 and p38-MAPK and JNK signal in the lumbar dorsal root ganglion. Behavioral test was performed to determine mechanical and cold sensitivity in a rat model. Our results showed that systemic injection of BTZ increased mechanical pain and cold sensitivity as compared with control animals. Data also showed that protein expression of TRPA1 and IL-6R was upregulated in the dorsal root ganglion of BTZ rats and blocking TRPA1 attenuated mechanical and cold sensitivity in control rats and BTZ rats. Notably, the inhibitory effect of blocking TRPA1 was smaller in BTZ rats than that in control rats. In addition, a blockade of IL- 6 signal attenuated intracellular p38-MAPK and JNK in the sensory neuron. This also decreased TRPA1 expression and alleviated mechanical hyperalgesia and cold hypersensitivity in BTZ rats. In conclusion, we revealed specific signaling pathways leading to neuropathic pain induced by chemotherapeutic BTZ, including IL-6-TRPA1, suggesting that blocking these signals is beneficial to alleviate neuropathic pain during BTZ intervention.
\end{abstract}

\section{Key words}

Bortezomib • Chemotherapeutics • TRPA1 • Neuro-inflammation - Peripheral neuropathy

\section{Corresponding authors}

D. Gao and H. Yu, Department of Otorhinolaryngology, The First Hospital of Jilin University, 71 Xinmin Street, Changchun, Jilin 130021, China. E-mail: gaodongmei64@aol.com

\section{Introduction}

One of the most common and distressing symptoms suffered by patients with progression of cancer is pain (Hanna et al. 2013). Cancer pain mainly arises from a tumor compressing or infiltrating tissue; from nerve and other changes caused by a hormone imbalance or immune response; and/or from treatments and diagnostic procedures (Pasetto et al. 2006, Hanna et al. 2013). Note that chemotherapy and radiotherapy may produce painful conditions persisting long after treatment has ended (Hoskin 2008, Portenoy 2011, Hanna et al. 2013). As a result, how to effectively manage cancer pain related to these therapies becomes an important issue for treatment and management of cancer patients in clinics.

Bortezomib (BTZ), a reversible inhibitor of the proteasome complex, is used predominantly as a chemotherapeutic agent for the treatment of multiple myeloma (Voorhees et al. 2003, Adams and Kauffman 2004). However, clinical and animal studies have demonstrated that dose-limiting painful peripheral neuropathy is one of the major adverse effects observed 
during BTZ therapy (Curran and McKeage 2009). Overall, treatment options for these abnormal sensations have been restricted, partly due to a poor understanding of the underlying mechanisms responsible for neuropathic pain induced by chemotherapeutic agents such as BTZ. Drugs preventing and/or treating the painful symptoms evoked by BTZ therapy are lacking. Therefore, it is significant to determine molecular mediators of BTZ-induced painful neuropathy to make therapeutic strategies for chemotherapeutic BTZ in patients with multiple myeloma.

Transient receptor potential ankyrin 1 (TRPA1) plays a functional role in regulating pain and neurogenic inflammation resulting from channel activation to a variety of compounds including pungent agents, irritant chemicals, reactive oxygen, and products of oxidative stress-induced lipid peroxidation (Bandell et al. 2004, Jordt et al. 2004, Bautista et al. 2005, Andersson et al. 2008, Sawada et al. 2008, Trevisan et al. 2013a, Trevisan et al. 2013c). TRPA1 appears in dorsal root ganglion (DRG) (Jordt et al. 2004) and is involved in development of mechanical hypersensitivity and painfully cold temperature (Story et al. 2003, Kwan et al. 2006). TRPA1 also mediates mechanical hyperalgesia and cold hypersensitivity induced by chemotherapeutic agents (Zhao et al. 2012, Trevisan et al. 2013b). Thus, in this report, we first postulated that sensory TRPA1 contributes to mechanical and cold hypersensitivity in rats that received BTZ. We hypothesized that injection of BTZ amplifies protein expression of TRPA1 in the DRG thereby resulting in mechanical pain and cold hypersensitivity. Blocking TRPA1 attenuates mechanical hyperalgesia and cold hypersensitivity observed in BTZ rats.

Pro-inflammatory cytokines, i.e. interleukin-6 (IL-6) play an important role in facilitating the development of neuropathic pain (Clark et al. 2013, Mika et al. 2013). In numerous neuropathic pain models, IL-6 and its receptor (IL-6R) expression in sensory nerves are upregulated following peripheral nerve injury (Miller et al. 2009). Local application of IL-6 induces persistent hyperalgesia and allodynia in naive rats (Carozzi et al. 2015). Inhibition of IL-6 signal attenuates mechanical hyperalgesia and allodynia (Miller et al. 2009). The application of chemotherapeutic drugs such as paclitaxel or vincristine also amplifies IL-6 levels (Carozzi et al. 2015). However, it is lacking whether IL-6 signal is engaged in BTZ-induced peripheral neuropathy. Thus, we examined the effects of IL-6 inhibition on protein expression of
TRPA1 in DRG as well as on mechanical and cold hypersensitivity in BTZ rats. We hypothesized that blocking IL-6 signal attenuates intracellular p38-MAPK and JNK in DRG and thereby decreases the protein expression levels of TRPA1 and further alleviates BTZ-induced mechanical hyperalgesia and cold hypersensitivity.

\section{Methods}

\section{Animal}

All animal protocols were in accordance with the guidelines of the International Association for the Study of Pain and approved by the Institutional Animal Care \& Use Committee of Jilin University. Male Sprague-Dawley rats (200-250 g) were housed in individual cages with free access to food and water and kept in a temperaturecontrolled room $\left(25^{\circ} \mathrm{C}\right)$ on a $12 / 12 \mathrm{~h}$ light/dark cycle.

\section{A model of neuropathic pain and administration of drugs}

BTZ $(0.4 \mathrm{mg} / \mathrm{kg}$ body weight; Haoran BioTech Co., Shanghai, China) was administrated intraperitoneally (i.p.) once daily for 5 consecutive days on the basis of previous reports (Zheng et al. 2012, Zhang et al. 2014). BTZ was first dissolved in $0.5 \%$ dimethyl sulfoxide (DMSO) and then diluted in $0.9 \%$ saline to obtain its concentration used in our experiments. Control animals received an equivalent volume of vehicle. After BTZ intervention, TRPA1 antagonist $\operatorname{HC} 030031$ (1, 3, $10 \mathrm{mg} / \mathrm{kg}$ body weight; Sigma, St. Louis, MO, USA) was administrated i.p. each day for the following 3 consecutive days. In the same fashion, SC144, an inhibitor to complexed IL-6R-gp130 (5, 10 and $20 \mathrm{mg} / \mathrm{kg}$ body weight; Sigma, St. Louis, MO, USA) was given i.p. each day for 3 consecutive days.

\section{Behavioral test}

To quantify the mechanical sensitivity of the hind paw, rats were placed in individual plastic boxes and allowed to acclimate for $>30 \mathrm{~min}$. Mechanical paw withdrawal threshold (PWT) of rat hind paw in response to the stimulation of von Frey filaments was determined. A series of calibrated von Frey filaments (ranging from 0.4 to $15 \mathrm{~g}$; BioSeb Co., Vitrolles, France) were applied perpendicularly to the plantar surface of the hind paw with an appropriate force to bend the filaments until paw withdrew. In the presence of a response, the filament of next lower force was applied. In the absence of a response, the filament of next greater force was applied. 
To avoid injury during tests, the cut-off strength of the von Frey filament was $15 \mathrm{~g}$. The tactile stimulus producing a $50 \%$ likelihood of withdrawal was determined using the "up-down" method (Chaplan et al. 1994). Each trial was repeated twice at 2 min intervals. The average of two measurements was calculated to obtain the mean value used as the force produced for a withdrawal response.

To examine cold sensitivity, Thermal Place Preference System (Coulburn Instruments) was used to perform the thermal place preference test in order to assess a cold avoidance behavior. Two connecting metal plates were surrounded by a plastic enclosure. The first plate was kept at neutral temperature $\left(25^{\circ} \mathrm{C}\right)$ and the second plate was kept at cold temperature $\left(12^{\circ} \mathrm{C}\right)$. The test was performed in darkness and each session lasted $3 \mathrm{~min}$. During the session, the rats were left free to explore both plates. The time spent on the cold plate during the entire session was recorded using an infrared camera connected to a computer to determine cold avoidance behavior. Thus, cold sensitivity was expressed as percentage time spent on the cold plate over $3 \mathrm{~min}$ [time on cold plate (seconds)/180 $\mathrm{s} \times \%$ ]. To better control behavior test, the rats were repeatedly placed on the apparatus with both plates held at room temperature $\left(25^{\circ} \mathrm{C}\right)$ during $3 \mathrm{~min}$ 2 days before the beginning of the experiment. Note that rats spent an equal amount of time on each plate under these conditions, suggesting that animals showed no place preference. Also, to avoid learning or any place preference unrelated to cold, the temperature of the plates were inverted between two consecutive sessions. Two trials were performed for each protocol and data were averaged. It is noted that the behavioral experiments were conducted blindly in this report.

\section{ELISA measurements}

All the tissues from individual rats were sampled for the analysis. In brief, DRG tissues (L4-L6) of the rats were removed. Total protein was then extracted by homogenizing the sample in ice-cold immunoprecipitation assay buffer with protease inhibitor cocktail kit (Promega Co. Madison, WI, USA). The lysates were centrifuged and the supernatants were collected for measurements of protein concentrations using a bicinchoninic acid assay reagent kit.

The levels of IL-6 were examined using an ELISA assay kit (Wuhan Fine Biotech Co.) according to the provided description. Briefly, polystyrene 96-well microtiter immunoplates were coated with affinity- purified rabbit anti-IL-6 antibodies. Parallel wells were coated with purified rabbit IgG for evaluation of nonspecific signal. After overnight incubation, plates were washed. The diluted samples and IL-6 standard solutions were distributed in each plate. The plates were washed and incubated with anti-IL- 6 galactosidase. The plates were washed and incubated with substrate solution. After incubation, the optical density was measured using an ELISA reader.

\section{Western blot analysis}

Briefly, DRG tissues (L4-L6) were removed and total protein was extracted. The lysates were centrifuged and the supernatants were collected. After being denatured, the supernatant samples containing $20 \mu \mathrm{g}$ of protein were loaded onto gels and electrically transferred to a polyvinylidene fluoride membrane. The membrane was incubated overnight with primary antibodies (diluted at 1:500): rabbit anti-IL-6R, anti-TRPA1, anti-p-p38MAPK/p38-MAPK and anti-p-JNK1/JNK1. The membranes were washed and incubated with an alkaline phosphatase conjugated anti-rabbit secondary antibody (1:1000). The primary and secondary antibodies were obtained from Abcam Co. or Antibodies online Com. The immunoreactive proteins were detected by enhanced chemiluminescence. The bands recognized by the primary antibody were visualized by exposure of the membrane onto an X-ray film. The membrane was stripped and incubated with anti- $\beta$-actin to show equal loading of the protein. The film was then scanned and the optical density of IL-6R/TRPA1/p-p38-MAPK/p38MAPK/p-JNK1/JNK1/ $\beta$-actin bands was analyzed using the Scion Image software.

\section{Statistical analysis}

All data were analyzed using a two-way repeated-measures analysis of variance. Values were presented as means \pm standard error of mean. As appropriate, Tukey's post hoc tests were used. For all analyses, differences were considered significant at $P<0.05$. All statistical analyses were performed by using SPSS for Windows version 13.0 (SPSS Inc.).

\section{Results}

\section{Basal levels of paw withdrawal threshold (PWT)}

Figure $1 \mathrm{~A}$ is a schematic diagram illustrating the timetable giving drugs and experimental protocols. Figure 1B demonstrates basal levels of PWT for 
5 consecutive days after BTZ was injected in rats. BTZ injection significantly decreased PWT as compared with saline control 3 days after its injection, suggesting neuropathic pain was developed in rats after administration of BTZ when the experiments were performed. Then, antagonists, HC030031 and SC144 were given to examine their effects on BTZ-induced pain.

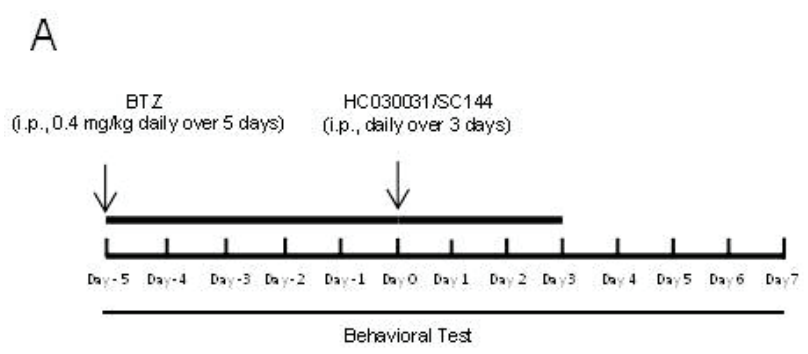

$\mathrm{B}$

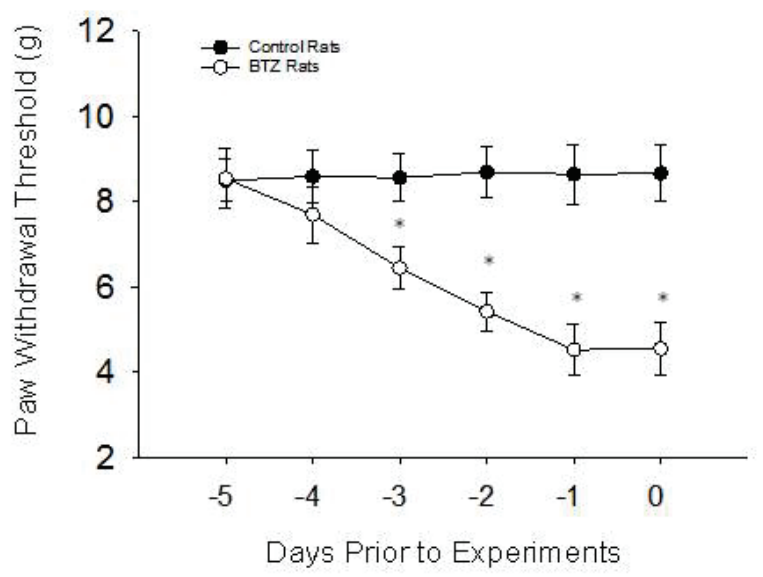

Fig. 1. (A) A schematic diagram showing the schedule giving drugs and experimental protocols. BTZ was injected [intraperitoneally (i.p.), daily, $0.4 \mathrm{mg} / \mathrm{kg}$ body weight]. The starting day is expressed as "day -5 ". An equivalent volume of saline was given in control animals. Then, HC030031 and SC144 were administered at the end of BTZ injection (day 0 ), which was also marked as day 0 as indicated in Figure 2 and Figure 3. (B) Basal paw withdrawal threshold (PWT). PWT is showed in control rats $(n=35)$ and rats following BTZ intervention $(n=46)$ prior to administration of $\mathrm{HC} 030031$ and $\mathrm{SC} 144$. $* P<0.05$ vs. saline control rats.

Effects of blocking TRPA1 on mechanical and cold sensitivity

Figure 2A-B show that PWT appeared to be smaller in BTZ rats than that in control rats for saline and three dosages of $\operatorname{HC} 030031(1,3,10 \mathrm{mg} / \mathrm{kg})$ at different time courses (0-7 days after HC030031). The figures also demonstrate that PWT was increased in a dose-dependent way after injection of $\operatorname{HC} 030031(1,3,10 \mathrm{mg} / \mathrm{kg})$ in control rats and BTZ rats. As $10 \mathrm{mg} / \mathrm{kg}$ of $\mathrm{HC} 030031$ was given, the effects were observed 1 day after its administration, and peaked at day 3 after its injection. The effects began to reduce after injection was discontinued. In addition, the percentage increase of PWT evoked by $\mathrm{HC} 030031$ was smaller in BTZ rats than that in control rats. i.e. as $10 \mathrm{mg} / \mathrm{kg}$ of $\mathrm{HC} 030031$ was given, PWT was increased by $59 \%$ in BTZ rats $(P<0.05$, control rats $/ \mathrm{n}=12$ vs. $\mathrm{BTZ}$ rats $/ \mathrm{n}=12$ ) and $72 \%$ in control rats 3 days after the first injection of $\mathrm{HC} 030031$.

In addition, Figure $2 \mathrm{C}$ shows that BTZ injection significantly decreased $\%$ time spent on the cold plate as compared with saline injection. Increases in cold sensitivity were greater in BTZ rats than these increases in control rats for saline and three dosages of $\mathrm{HC} 030031$. This figure further shows that blocking TRPA1 with HC030031 $(10 \mathrm{mg} / \mathrm{kg})$ for three day attenuated cold sensitivity to a greater degree in control rats $(\%$ time increased by $62 \%, \mathrm{n}=12)$ than in BTZ rats ( $\%$ time increased by $42 \%, \mathrm{n}=12, P<0.05$ vs. control rats).

\section{Effects of blocking IL-6 signal on mechanical and cold} sensitivity

Figure $3 \mathrm{~A}$ and B show that PWT appeared to be smaller in BTZ rats than that in control rats for saline and three dosages of SC144 $(5,10,20 \mathrm{mg} / \mathrm{kg})$ at different time courses. We further examined the role played by inhibition of IL-6 in mechanical and cold sensitivity. The figures also show that injection of SC144 attenuated mechanical sensitivity in BTZ rats and control rats as compared with saline injection. The inhibitory effects of SC144 on mechanical sensitivity appeared in a dosedependent way. When $20 \mathrm{mg} / \mathrm{kg}$ of SC144 was injected, the effects were observed 1 day after its administration and peaked at day 3 . The effects began to reduce after its injection was discontinued. The effects were smaller in $\mathrm{BTZ}$ rats than in control rats $(P<0.05, \mathrm{BTZ}$ vs. controls). In addition, Figure $3 \mathrm{C}$ shows that cold sensitivity was increased in BTZ rats as compared with saline control rats and rats with three dosages of SC144. Inhibition of IL-6 signal with SC144 attenuated cold sensitivity to a greater degree in control rats than in BTZ rats $(P<0.05$, BTZ vs. controls; $\mathrm{n}=12$ in each group).

\section{Effects of blocking IL-6 on TRPA1 signal leading to} neuropathic pain

The effects of IL-6 on TRPA1 signal pathway were examined in additional groups. In this experiment, DRG tissues were removed three days after the beginning 
injection of SC144. First, the levels of IL-6 and expression of IL-6R were examined in the DRG of control rats and BTZ rats. Figure 4A demonstrates that BTZ administration amplified the levels of IL- 6 in DRG as compared with control rats $(P<0.05$, BTZ rats vs. control rats, $\mathrm{n}=12$ in each group). BTZ also increased protein expression of IL-6R as compared with control rats $(P<0.05, \mathrm{BTZ}$ rats vs. control rats, $\mathrm{n}=6-8$ in each group). In addition, Figure 4B-D demonstrate that TRPA1 was upregulated by BTZ and SC144 attenuated upregulation of TRPA1 induced by BTZ $(P<0.05$ vs. BTZ without $\mathrm{SC144)}$. Moreover, intracellular signal pathways of DRG neurons, namely, p38-MAPK and JNK, were examined (Fig. 4B-D). BTZ upregulated phosphorylated p38-MAPK and JNK and these amplifications were inhibited by administration of SC144. It is noted that total protein levels of p38-MAPK and JNK were not increased significantly by BTZ, but the ratio of phosphorylated p38-MAPK and JNK and total phosphorylated p38-MAPK and JNK was significantly increased by BTZ.

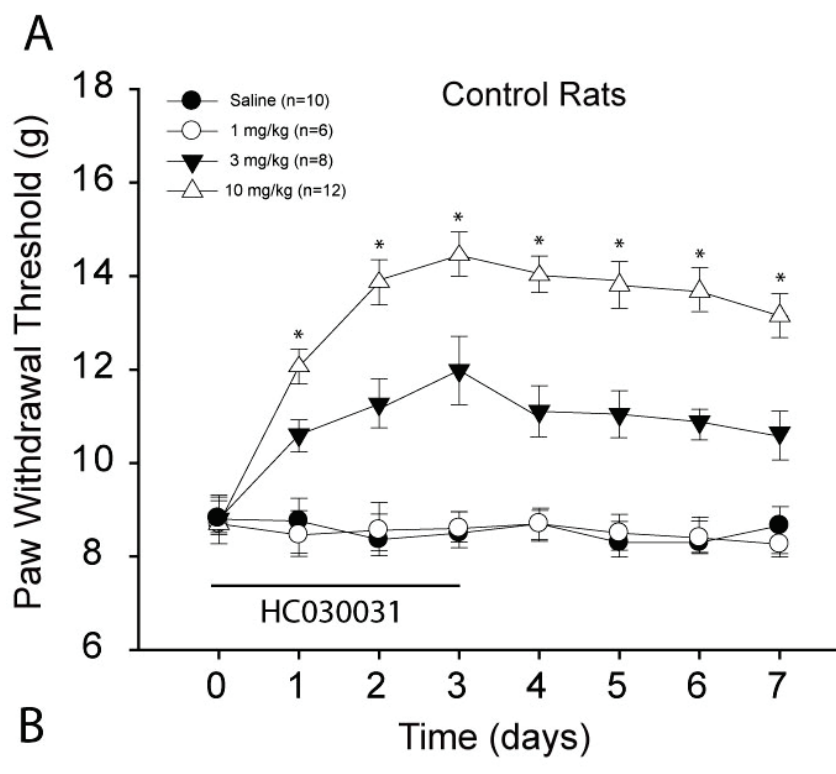

\section{C}

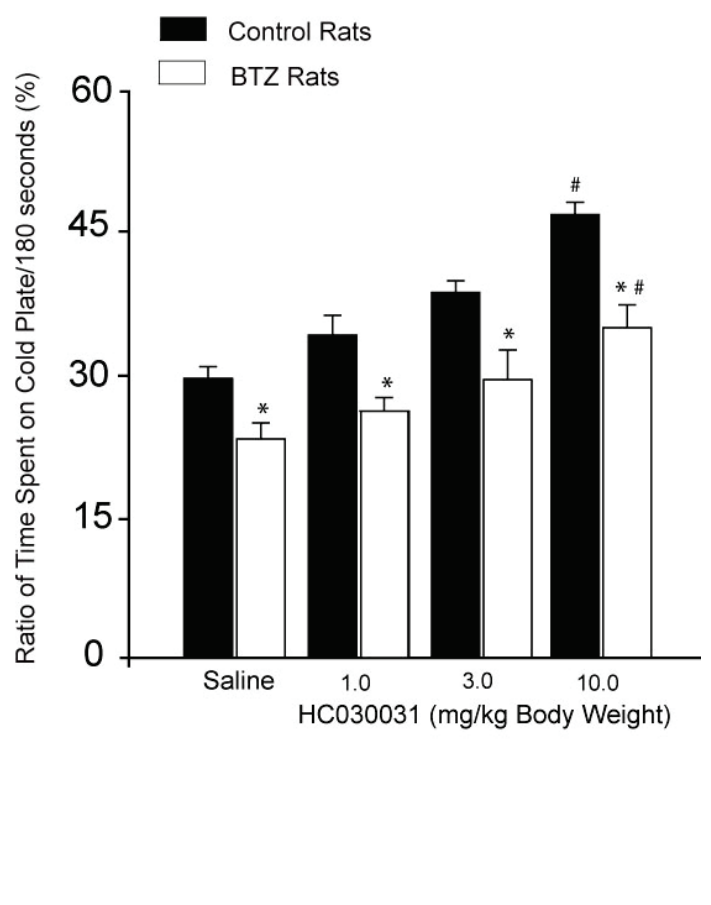

Fig. 2. Effects of blocking TRPA1 on mechanical and cold sensitivity. TRPA1 was blocked by administration of HC030031 (1, 3, $10 \mathrm{mg} / \mathrm{kg}$ body weight; i.p. each day over 3 consecutive days). Paw withdrawal threshold (PWT, g) and cold sensitivity expressed as time spent on the cold plate (\%) were examined in control rats and BTZ rats. (A, B) PWT was smaller in BTZ rats than that in control rats for saline and three dosages of HC030031 at different time courses. As compared with saline injection, HC030031 increased PWT in control rats and BTZ rats. The increases of PWT evoked by attenuation of TRPA1 were smaller in BTZ rats than these in control rats. $* P<0.05$ vs. saline control and other dosages. The number of rats in each group was shown on the figure. (C) showing that time spent on the cold plate was less in BTZ rats than in control rats for saline and three dosages of HC030031. HC030031 elevated \% time spent on the cold plates in control rats and BTZ rats 3 days after its injection as compared with saline injection. The effects of HC030031 on cold sensitivity were smaller in BTZ rats than these in control rats. $* P<0.05, \mathrm{BTZ}$ rats vs. control rats for saline and three dosages of HC030031 three days after injection of HC030031. ${ }^{\#} P<0.05$ vs. saline control and other dosages. 

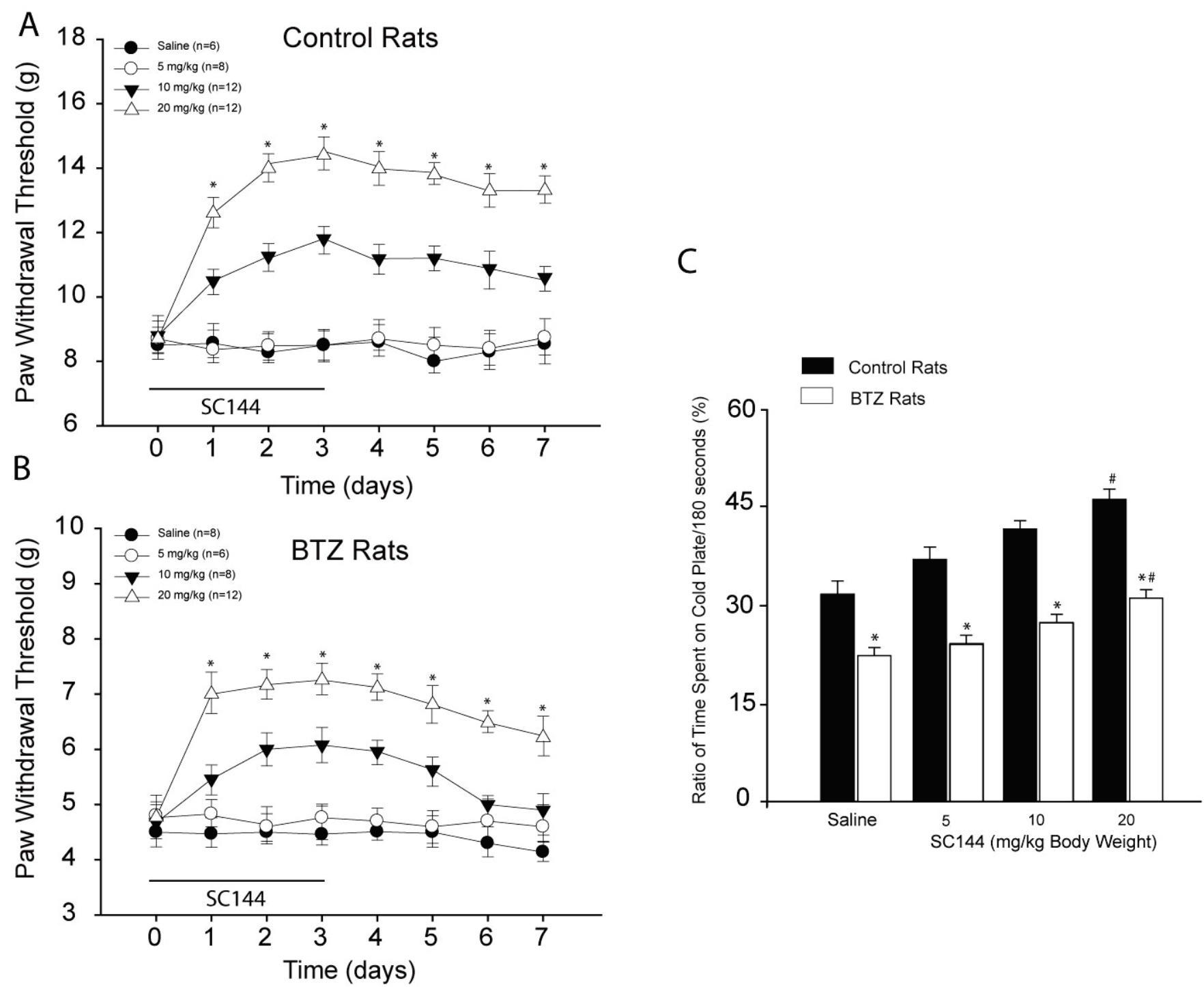

Fig. 3. Effects of blocking IL-6 signal on mechanical and cold sensitivity. IL-6R was inhibited by SC144 (5, 10, $20 \mathrm{mg} / \mathrm{kg}$ body weight; i.p. each day over 3 consecutive days). Paw withdrawal threshold (PWT) and cold sensitivity expressed as time spent on the cold plate (\%) were examined in control rats and BTZ rats. (A, B) PWT was smaller in BTZ rats than in control rats for saline and three dosages of SC144 at different time courses. SC144 increased PWT in control rats and BTZ rats as compared with saline control, but the amplitude of PWT increases evoked by SC144 was smaller in BTZ rats than that in control rats. $* P<0.05$ vs. saline and other dosages. The number of rats in each group was shown on the figure. (C) showing that time spent on the cold plate was less in BTZ rats than in control rats for saline and three dosages of SC144. SC144 elevated \% time spent on the cold plates in control rats and BTZ rats 3 days after its injection. The effects of SC144 on cold sensitivity were smaller in BTZ rats than these in control rats. $* P<0.05$, BTZ rats vs. control rats for saline and three dosages of SC144 three days after its injection. ${ }^{\#} P<0.05$ vs. saline and other dosages.

\section{Discussion}

Results of the current study showed that systemic injection of BTZ increased mechanical pain and cold sensitivity accompanied with upregulation of IL-6R and TRPA1 in the DRG. Blocking IL-6 signal decreased TRPA1 expression and alleviated mechanical hyperalgesia and cold hypersensitivity via intracellular p38-MAPK and JNK in BTZ rats.

Prior studies have shown that injection of BTZ can induce neuropathic pain including mechanical hyperalgesia and cold hypersensitivity in rats after initiation of the chemotherapy regimen (Chiorazzi et al. 2013, Trevisan et al. 2013b, Zhang et al. 2014, Li et al. 2016). Of note, the signs of neuropathy start with BTZ therapy. These abnormalities were ablated several days after discontinuation of BTZ (Chiorazzi et al. 2013, Zhang et al. 2014, Li et al. 2016). Thus, we used this well-established rat model to study the mechanisms of neuropathic pain induced by chemotherapeutic BTZ in this report.

We examined the effects of blocking TRPA1 
and IL-6 signal in sensory nerves on mechanical hyperalgesia and cold hypersensitivity in following administration of BTZ. Consistent with the previous findings (Cavaletti et al. 2007, Chiorazzi et al. 2013, Trevisan et al. 2013b, Zhang et al. 2014, Li et al. 2016), in this study we observed that BTZ decreased PWT and evoked less time (\%) spent on the cold plate after its injection. Our results further demonstrated that injection of TRPA1 antagonist significantly increased PWT and $\%$ time spent on the cold plate in BTZ rats. It is noted that the effects of HC030031 were also observed in control rats, but the effects of blocking TRPA1 were smaller in
BTZ rats as compared with control animals. Likewise, blocking IL-6 signal had the less effects on PWT and $\%$ time spent on the cold plate in BTZ rats. In relation to this result, our data also showed that BTZ led to upregulation of TRPA1 and IL-6R in the DRG. Upregulation of TRPA1 and IL-6R in the DRG of BTZ rats led to mechanical hyperalgesia and cold hypersensitivity. The same dosage of HC030031 and SC144 had the less inhibitory effects on mechanical hyperalgesia and cold hypersensitivity in BTZ rats due to upregulation of TRPA1 and IL-6R.
A
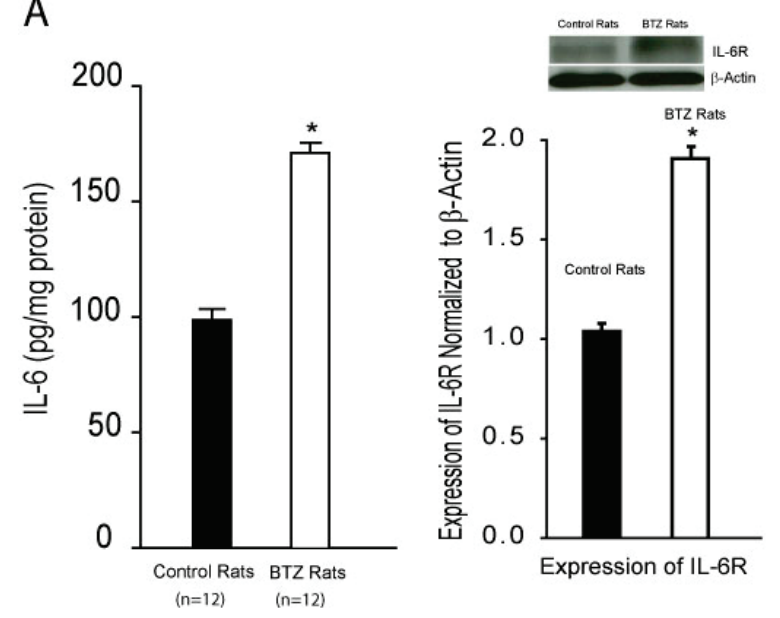

B
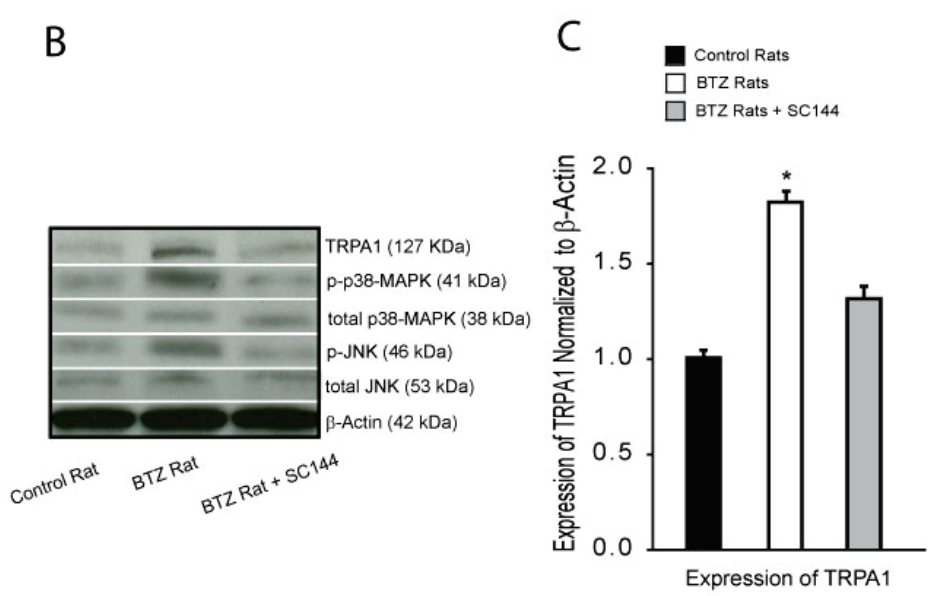

D
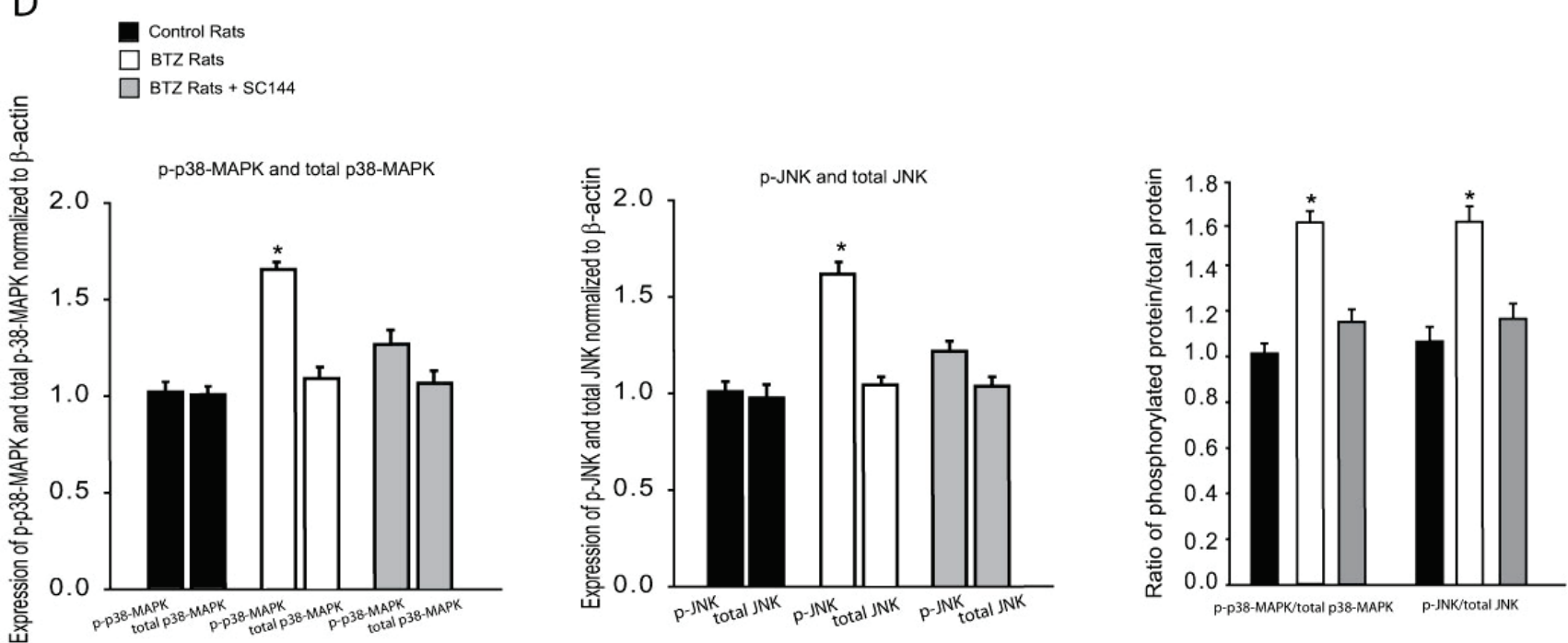

Fig. 4. Effects of IL-6 inhibition on TRPA1 signal pathways leading to neuropathic pain. SC144 (20 mg/kg body weight, i.p. each day for 3 consecutive days) was given to inhibit IL- 6 signal pathways. DRG tissues were removed for examination expression of TRPA1 and intracellular signal three days after the beginning injection of SC144. (A) The levels of IL-6 and IL-6R expression were amplified in the DRG tissues of BTZ rats. $* P<0.05, \mathrm{BTZ}$ rats vs. control rats. $\mathrm{n}=12$ in each group for measurement of IL-6. $\mathrm{n}=6$ in control rats and $\mathrm{n}=8$ in BTZ rats for western blot analysis. (B) Typical bands represented for averaged data in C\&D. (C, D) Averaged data show that BTZ increased the protein levels of TRPA1 and intracellular signal p-p38-MAPK and p-JNK (phosphorylated form) in the DRG as compared with controls. Furthermore, administration of SC144 attenuated amplification of TRPA1 and these signal pathways in BTZ rats. Total protein levels of p38-MAPK and JNK were not elevated significantly by BTZ, but the ratio of phosphorylated p38-MAPK and JNK versus total p38-MAPK and JNK was increased by BTZ. * $P<0.05$ vs. control rats and BTZ rats with SC144. $\mathrm{n}=8-12$ in each group. 
IL-6 complexes with membrane-bound or soluble IL6R to activate cells expressing the signal transducer glycoprotein (gp130) (Hoge et al. 2013, Wolf et al. 2014, Ruwanpura et al. 2016). Most cells are devoid of membrane-bound IL-6R and are thus unresponsive to IL-6; however, they can still react to IL-6 complexed with a soluble form of the IL-6R (sIL-6R) to activate gp130, a pathway called "trans-signaling" (RoseJohn and Heinrich 1994). Thus, in the current study we used SC144, a gp130 inhibitor, to block IL-6-mediated signal transduction in order to examine engagement of the IL-6R in mechanical hyperalgesia induced by BTZ. Our data showed that systemic administration of SC144 significantly amplified PWT and \% time spent on the cold plate in BTZ animals. Interestingly, SC144 attenuated expression of TRPA1 in the DRG of BTZ rats, suggesting the role played by IL- 6 in modifying TRPA1 under BTZ treatment. Intracellular signals p38-MAPK and p-JNK are also engaged in the effects of IL-6. Accordingly, our data indicate that BTZ activates IL-6 signal, which subsequently amplifies expression of TRPA1 in the DRG via intracellular p38-MAPK and JNK signals thereby resulting in mechanical hyperalgesia and cold hypersensitivity.

TRPA1 has a functional role in regulating pain and neurogenic inflammation resulting from channel activation to a variety of compounds (Bandell et al. 2004, Jordt et al. 2004, Bautista et al. 2005, Andersson et al. 2008, Sawada et al. 2008, Trevisan et al. 2013a, Trevisan et al. 2013b, Trevisan et al. 2013c). TRPA1 is expressed in DRG neurons (Jordt et al. 2004) and is engaged in development of mechanical hypersensitivity and painfully cold temperatures (Story et al. 2003, Kwan et al. 2006). Additional studies further indicate that TRPA1 mediates mechanical and cold hypersensitivity induced by chemotherapeutic agents (Zhao et al. 2012, Trevisan et al. 2013b, Nassini et al. 2015). In particular, our current study suggests that TRPA1 plays a role in regulating BTZ-evoked neuropathic pain. IL-6 is a signal activating TRPA1 because inhibition of IL- 6 attenuates BTZ-evoked upregulation of TRPA1 and mechanical hyperalgesia and cold hypersensitivity.

Pro-inflammatory IL-6, IL-1 $\beta$ and TNF- $\alpha$ have been reported to be involved in BTZ-induced neuropathic pain (Zhang et al. 2014, Li et al. 2016). For example, IL-6 was elevated in the circulation of patients with BTZ treatment and its levels were correlated with the appearance of BTZ-induced neuropathic pain (Mangiacavalli et al. 2010). IL-1 $\beta$ and IL-6 were elevated in the DRG of rats with injection of BTZ (Ale et al. 2014). BTZ treatment induced upregulation of the mRNA and protein levels of IL- $1 \beta$ in the astrocytes of the spinal dorsal horn of rats and this also increased the phosphorylation of JNK ( $\mathrm{Li}$ et al. 2016). Inhibition of IL-1 $\beta$ and TNF- $\alpha$ alleviated JNK activation and lessened mechanical allodynia induced by BTZ (Zhang et al. 2014, Li et al. 2016).

IL-6 activates multiple signaling pathways, including the p38-MAPK and JNK pathways (Miller et al. 2009), which are recognized as important regulators of inflammatory pain. The prior study has reported that IL-6 amplified TRPA1 via intracellular p38-MAPK signal (El Karim et al. 2015). On the basis of those results, in the current study we designed our in vivo experiments. Indeed, we observed that inhibition of IL-6 signal by SC144 decreased expression of TRPA1, p38-MAPK and JNK in the DRG of BTZ rats and this further attenuated neuropathic pain induced by BTZ. Nonetheless, our results showed that p38-MAPK and JNK signal are stimulated by BTZ and likely mediate upregulation of TRPA1 in sensory nerves. We demonstrated, for the first time, a novel mechanism by which IL-6 contributes to enhanced TRPA1 likely via p38-MAPK and JNK, which are involved in mechanical hyperalgesia and cold hypersensitivity induced by BTZ.

TRPA1 receptors are well known to be essential in the transduction of mechanical, cold and chemical stimuli (Kwan et al. 2006, Kerstein et al. 2009, Trevisan et al. 2013a, Trevisan et al. 2013c, Zappia et al. 2017). In TRPA1 knockout animals or animals after pharmacological TRPA1 ablation, pain responses to mechanical, cold and chemical stimuli appear to be decreased (Kwan et al. 2006, Kerstein et al. 2009, Trevisan et al. 2013a, Trevisan et al. 2013c, Zappia et al. 2017). In the current study, our data showed that both HC030031 and SC144 significantly reduced BTZ-induced mechanical hyperalgesia and cold hypersensitivity; however a remarkably larger effect of these antagonists was observed in control animals. This suggests that both TRPA1 and IL-6 signaling are also involved in the transduction of mechanical and cold sensitivity in control animals. Due to a lower inhibitory efficacy in BTZ group, these results indicate that it is likely that TRPA1 and IL-6R expression and/activity are amplified in the sensory nervous system. Indeed, our data showed that protein expression of TRPA1 and IL-6R was upregulated in the DRG of BTZ rats. One may think that these results indicate that both TRPA1 and IL- 6 signaling 
have less significant importance in the BTZ models compared to the control situation. Also, this may be due to an involvement of other mechanisms that likely participate in the development of the BTZ-induced hypersensitivity, not only due to upregulation of TRPA1 and IL-6R.

In conclusion, the protein expression of TRPA1 in peripheral sensory nerves are upregulated by injection of BTZ; inhibition of TRPA1 and IL- 6 signal antagonizes mechanical hyperalgesia and cold hypersensitivity during BTZ intervention; and TRPA1 play a role in IL-6 engagement of BTZ-induced neuropathic pain via intracellular p38-MAPK and JNK signal. Results of this study suggest the mechanisms responsible for
BTZ-induced neuropathic pain. In particular, targeting one or more of these signaling molecules involved in activation of IL-6 and TRPA1 evoked by BTZ may present new opportunities for treatment and management of neuropathic pain in patients with multiple myeloma during BTZ chemotherapeutics.

\section{Conflict of Interest}

There is no conflict of interest.

\section{Acknowledgements}

The authors thank all the people who participated in this study.

\section{References}

ADAMS J, KAUFFMAN M: Development of the proteasome inhibitor velcade (bortezomib). Cancer Invest 22: 304-311, 2004.

ALE A, BRUNA J, MORELL M, VAN DE VELDE H, MONBALIU J, NAVARRO X, UDINA E: Treatment with anti-TNF alpha protects against the neuropathy induced by the proteasome inhibitor bortezomib in a mouse model. Exp Neurol 253: 165-173, 2014.

ANDERSSON DA, GENTRY C, MOSS S, BEVAN S: Transient receptor potential A1 is a sensory receptor for multiple products of oxidative stress. $J$ Neurosci 28: 2485-2494, 2008.

BANDELL M, STORY GM, HWANG SW, VISWANATH V, EID SR, PETRUS MJ, EARLEY TJ, PATAPOUTIAN A: Noxious cold ion channel TRPA1 is activated by pungent compounds and bradykinin. Neuron 41: 849-857, 2004.

BAUTISTA DM, MOVAHED P, HINMAN A, AXELSSON HE, STERNER O, HOGESTATT ED, JULIUS D, JORDT SE, ZYGMUNT PM: Pungent products from garlic activate the sensory ion channel TRPA1. Proc Natl Acad Sci U S A 102: 12248-12252, 2005.

CAROZZI VA, CANTA A, CHIORAZZI A: Chemotherapy-induced peripheral neuropathy: What do we know about mechanisms? Neurosci Lett 596: 90-107, 2015.

CAVALETTI G, GILARDINI A, CANTA A, RIGAMONTI L, RODRIGUEZ-MENENDEZ V, CERESA C, MARMIROLI P, BOSSI M, OGGIONI N, D'INCALCI M, DE COSTER R: Bortezomib-induced peripheral neurotoxicity: a neurophysiological and pathological study in the rat. Exp Neurol 204: 317-325, 2007.

CHAPLAN SR, BACH FW, POGREL JW, CHUNG JM, YAKSH TL: Quantitative assessment of tactile allodynia in the rat paw. J Neurosci Methods 53: 55-63, 1994.

CHIORAZZI A, CANTA A, MEREGALli C, CAROZZI V, SALA B, OGGIONI N, MONBALIU J, VAN DE VELDE H, CAVALETTI G: Antibody against tumor necrosis factor-alpha reduces bortezomib-induced allodynia in a rat model. Anticancer Res 33: 5453-5459, 2013.

CLARK AK, OLD EA, MALCANGIO M: Neuropathic pain and cytokines: current perspectives. J Pain Res 6: 803-814, 2013.

CURRAN MP, MCKEAGE K: Bortezomib: a review of its use in patients with multiple myeloma. Drugs 69: 859-888, 2009.

EL KARIM I, MCCRUDDEN MT, LINDEN GJ, ABDULLAH H, CURTIS TM, MCGAHON M, ABOUT I, IRWIN C, LUNDY FT: TNF-alpha-induced p38MAPK activation regulates TRPA1 and TRPV4 activity in odontoblastlike cells. Am J Pathol 185: 2994-3002, 2015.

HANNA M, ZYLICZ Z: Cancer Pain. Springer-Verlag, London, 2013, 286 p. 
HOGE J, YAN I, JANNER N, SCHUMACHER V, CHALARIS A, STEINMETZ OM, ENGEL DR, SCHELLER J, ROSE-JOHN S, MITTRUCKER HW: IL-6 controls the innate immune response against Listeria monocytogenes via classical IL-6 signaling. J Immunol 190: 703-711, 2013.

HOSKIN PJ: Radiotherapy. In: Clinical Pain Management: Cancer Pain. SYKES N, BENNETT MI, YUAN C-S (eds), Hodder Arnold, London, 2008, pp 251-255.

JORDT SE, BAUTISTA DM, CHUANG HH, MCKEMY DD, ZYGMUNT PM, HOGESTATT ED, MENG ID, JULIUS D: Mustard oils and cannabinoids excite sensory nerve fibres through the TRP channel ANKTM1. Nature 427: 260-265, 2004.

KERSTEIN PC, DEL CAMINO D, MORAN MM, STUCKY CL: Pharmacological blockade of TRPA1 inhibits mechanical firing in nociceptors. Mol Pain 5: 19, 2009.

KWAN KY, ALLCHORNE AJ, VOLLRATH MA, CHRISTENSEN AP, ZHANG DS, WOOLF CJ, COREY DP: TRPA1 contributes to cold, mechanical, and chemical nociception but is not essential for hair-cell transduction. Neuron 50: 277-289, 2006.

LI ZY, ZHANG YP, ZHANG J, ZHANG SB, LI D, HUANG ZZ, XIN WJ: The possible involvement of JNK activation in the spinal dorsal horn in bortezomib-induced allodynia: the role of TNF-alpha and IL-1beta. $J$ Anesthesia 30: 55-63, 2016.

MANGIACAVALLI S, CORSO A, DE AMICI M, VARETTONI M, ALFONSI E, LOZZA A, LAZZARINO M: Emergent T-helper 2 profile with high interleukin-6 levels correlates with the appearance of bortezomibinduced neuropathic pain. Br J Haematol 149: 916-918, 2010.

MIKA J, ZYCHOWSKA M, POPIOLEK-BARCZYK K, ROJEWSKA E, PRZEWLOCKA B: Importance of glial activation in neuropathic pain. Eur J Pharmacol 716: 106-119, 2013.

MILLER RJ, JUNG H, BHANGOO S, WHITE FA: Cytokine and chemokine regulation of sensory neuron function. In: Handbook of Experimental Pharmacology. CANNING BJ, SPINA DV (eds), Springer: Berlin Heidelberg, 2009, pp 417-449.

NASSINI R, FUSI C, MATERAZZI S, COPPI E, TUCCINARDI T, MARONE IM, DE LOGU F, PRETI D, TONELLO R, CHIARUGI A, PATACCHINI R, GEPPETTI P, BENEMEI S: The TRPA1 channel mediates the analgesic action of dipyrone and pyrazolone derivatives. Br J Pharmacol 172: 3397-3411, 2015.

PASETTO LM, D'ANDREA MR, ROSSI E, MONFARDINI S: Oxaliplatin-related neurotoxicity: How and why? Crit Rev Oncol Hematol 59: 159-168, 2006.

PORTENOY RK: Treatment of cancer pain. Lancet 377: 2236-2247, 2011.

ROSE-JOHN S, HEINRICH PC: Soluble receptors for cytokines and growth factors: generation and biological function. Biochem J 300: 281-290, 1994.

RUWANPURA SM, MCLEOD L, DOUSHA LF, SEOW HJ, ALHAYYANI S, TATE MD, DESWAERTE V, BROOKS GD, BOZINOVSKI S, MACDONALD M, GARBERS C, KING PT, BARDIN PG, VLAHOS R, ROSE-JOHN S, ANDERSON GP, JENKINS BJ: Therapeutic targeting of the IL-6 trans-signaling/mechanistic target of rapamycin complex 1 axis in pulmonary emphysema. Am J Resp Crit Care Med 194: 1494-1505, 2016.

SAWADA Y, HOSOKAWA H, MATSUMURA K, KOBAYASHI S: Activation of transient receptor potential ankyrin 1 by hydrogen peroxide. Eur J Neurosci 27: 1131-1142, 2008.

STORY GM, PEIER AM, REEVE AJ, EID SR, MOSBACHER J, HRICIK TR, EARLEY TJ, HERGARDEN AC, ANDERSSON DA, HWANG SW, MCINTYRE P, JEGLA T, BEVAN S, PATAPOUTIAN A: ANKTM1, a TRP-like channel expressed in nociceptive neurons, is activated by cold temperatures. Cell 112: 819-829, 2003.

TREVISAN G, HOFFMEISTER C, ROSSATO MF, OLIVEIRA SM, SILVA MA, INEU RP, GUERRA GP, MATERAZZI S, FUSI C, NASSINI R, GEPPETTI P, FERREIRA J: Transient receptor potential ankyrin 1 receptor stimulation by hydrogen peroxide is critical to trigger pain during monosodium urate-induced inflammation in rodents. Arthritis Rheum 65: 2984-2995, 2013 a.

TREVISAN G, MATERAZZI S, FUSI C, ALTOMARE A, ALDINI G, LODOVICI M, PATACCHINI R, GEPPETTI P, NASSINI R: Novel therapeutic strategy to prevent chemotherapy-induced persistent sensory neuropathy by TRPA1 blockade. Cancer Res 73: 3120-3131, 2013b. 
TREVISAN G, ROSSATO MF, HOFFMEISTER C, OLIVEIRA SM, SILVA CR, MATHEUS FC, MELLO GC, ANTUNES E, PREDIGER RD, FERREIRA J: Mechanisms involved in abdominal nociception induced by either TRPV1 or TRPA1 stimulation of rat peritoneum. Eur J Pharmacol 714: 332-344, 2013c.

VOORHEES PM, DEES EC, O'NEIL B, ORLOWSKI RZ: The proteasome as a target for cancer therapy. Clin Cancer Res 9: 6316-6325, 2003.

WOLF J, ROSE-JOHN S, GARBERS C: Interleukin-6 and its receptors: a highly regulated and dynamic system. Cytokine 70: 11-20, 2014.

ZAPPIA KJ, O'HARA CL, MOEHRING F, KWAN KY, STUCKY CL: Sensory neuron-specific deletion of TRPA1 results in mechanical cutaneous sensory deficits. eNeuro 4: pii: ENEURO.0069-16.2017, 2017.

ZHANG J, SU YM, LI D, CUI Y, HUANG ZZ, WEI JY, XUE Z, PANG RP, LIU XG, XIN WJ: TNF-alpha-mediated JNK activation in the dorsal root ganglion neurons contributes to bortezomib-induced peripheral neuropathy. Brain Behav Immun 38: 185-191, 2014.

ZHAO M, ISAMI K, NAKAMURA S, SHIRAKAWA H, NAKAGAWA T, KANEKO S: Acute cold hypersensitivity characteristically induced by oxaliplatin is caused by the enhanced responsiveness of TRPA1 in mice. Mol Pain 8: 55, 2012.

ZHENG H, XIAO WH, BENNETT GJ: Mitotoxicity and bortezomib-induced chronic painful peripheral neuropathy. Exp Neurol 238: 225-234, 2012. 